\title{
Atenção ao parto por enfermeiros na Zona Leste do município de São Paulo
}

\author{
Labor care delivered by nurses in the East Zone of the municipality of São Paulo, SP, Brazil \\ Atención al parto por enfermeors en la Zona Leste de la cuidad de São Paulo, SP, Brasil
}

\author{
Nádia Zanon Narchi' \\ 'Universidade de São Paulo. Escola de Artes. Ciências e Humanidades. Curso de Obstetrícia. Grupo de Pesquisa Qualidade na \\ Atenção e Educação na Saúde da Mulher e do Recém-Nascido. São Paulo, SP
}

Submissão: 18/03/2009

Aprovação: 02/07/2009

\section{RESUMO}

Com o objetivo de analisar a atenção ao parto pelos enfermeiros dos seis hospitais públicos da zona leste do município de São Paulo foi realizado estudo descritivo-exploratório, de outubro de 2006 a junho de 2007, com dirigentes de enfermagem e 38 enfermeiros Que responderam Questionário, indicando a frequência com Que realizavam atividades relativas à assistência, bem como os obstáculos ao seu trabalho. Os resultados mostraram Que os enfermeiros não dispunham de meios para operacionalizar o cuidado devido às barreiras institucionais e organizacionais com Que se defrontavam. Conclui-se Que para Que possam exercer plenamente sua profissão na atenção ao parto os enfermeiros devem contar com estruturas mais favoráveis e fortalecer sua identidade profissional, o Que pressupõe maior Qualificação e poder.

Descritores: Enfermagem obstétrica; Políticas públicas; Mortalidade materna; Mortalidade perinatal; Humanização da assistência.

\begin{abstract}
The aim of this study was to analyze delivery care by nurses in six public hospitals of the East zone in the municipality of São Paulo. A descriptive-exploratory study was carried out from October 2006 to June 2007, with nursing leaders and 38 nurses who answered the Questionnaire, indicating frequency they performed delivery care related activities, as well as any possible obstacles or difficulties to exercise their functions. The results showed that nurses had no means to operationalize their work due to institutional and organization barriers that they had to deal with. It was concluded that to completely perform their work regarding delivery care, nurses must count with more favorable structures and to strengthen their professional identity, thus assuming greater Qualification and power.
\end{abstract}

Descriptors: Obstetrical nursing; Public policies; Maternal mortality; Perinatal mortality; Humanization of assistance.

\section{RESUMEN}

Con el objetivo de analizar la atención al parto por los enfermeros de los seis hospitales públicos de la zona leste, de la ciudad de São Paulo, se hizo realizar un estudio descriptivo-exploratorio, de octubre de 2006 a junio de 2007, con dirigentes de enfermería y 38 enfermeros Que respondieron al cuestionario, indicando la frecuencia con que habían realizado actividades relativas a la atención al parto, bien como los obstáculos para prestar esta asistencia. Los resultados demostraron que los enfermeros no disponían de medios para operacionalizar su trabajo debido a las barreras institucionales y organizacionales con Que se depararan. Concluyese Que para Que puedan ejercer plenamente su profesión en la atención al parto los enfermeros deben poder contar con estructuras favorables y fortalecer su identidad profesional, lo Que presupone mayor calificación y poder.

Descriptores: Enfermería obstétrica; Políticas públicas; Mortalidad materna; Mortalidad perinatal; Humanización de la atención. 


\section{INTRODUÇÃO}

A Qualificação dos profissionais médicos e não-médicos para manejar gestação, parto e pós-parto é uma das estratégias Que comprovadamente produz redução da morbi-mortalidade materna e perinatal ${ }^{(1,2)}$.

No Que se refere aos não-médicos e tendo em vista a relevância da Qualificação de enfermeiras e/ou obstetrizes, a Confederação Internacional de Obstetrizes (ICM), em conjunto com a Organização Mundial da Saúde (OMS) e a Federação Internacional de Ginecologia e Obstetrícia (FIGO), estabeleceu as competências essenciais para o exercício básico da obstetrícia, ou seja, Que conhecimentos e habilidades os profissionais devem possuir para assistir a mulher de forma efetiva e baseada nas melhores e mais atualizadas evidências científicas em todas as fases do ciclo gravídico-puerperal, o Que inclui realização de partos de baixo risco, prestação de assistência ao recém-nascido e ao lactente, prevenção e detecção de intercorrências e execução de medidas de urgência na ausência de ajuda médica ${ }^{(2,3)}$.

Conforme orienta a $\mathrm{OMS}^{(2)}$, todos os implicados no sistema de saúde, incluindo o aparelho formador, devem reconhecer essas competências e proporcionar condições para Que sejam colocadas em prática, a bem das mulheres e dos indicadores de Qualidade assistencial. Nesse sentido, faz-se importante ressaltar a existência de diversos trabalhos mostrando Que os resultados assistenciais materno-infantis são melhores Quando as mulheres são atendidas por enfermeiras e/ou obstetrizes ${ }^{(4-10)}$.

A esse respeito e no Que tange ao Brasil, somente a partir de 1998 dispositivos legais, programas e pactos promulgados pelo governo destacaram o papel das enfermeiras, especialmente as especialistas em Obstetrícia, na promoção da maternidade segura. A partir de então muitas medidas foram tomadas, inclusive aporte financeiro para a formação de maior Quantitativo de enfermeiras obstétricas, assim como o desenvolvimento de outras estratégias como estímulo e apoio à criação dos centros de parto normal intra ou extra-hospitalares, investimento na Qualificação das maternidades, capacitação e educação permanente dos envolvidos na atenção à saúde materna e perinatal, entre outras.

Tendo em vista essa necessidade de maior número de profissionais Qualificados técnica e cientificamente para auxiliar a transformar o modelo de atenção à saúde materna e perinatal, o Que necessariamente implica na redução da morbi-mortalidade na área, foi implantado pela Universidade de São Paulo, em 2005, o Curso de Graduação em Obstetrícia, cuja perspectiva é formar obstetrizes Que juntem esforços com as enfermeiras obstétricas na melhoria substantiva da atenção materno-infantil.

Pela importância da participação dessas profissionais na promoção da maternidade segura e considerando Que devam possuir condições para o exercício de sua profissão, define-se como objeto deste estudo a atenção ao parto pelos enfermeiros na Zona Leste (ZL) do município de São Paulo Que, segundo dados de 2006 (1I), apresentou indicadores de má Qualidade da atenção materno-infantil.

\section{OBJETIVO}

Esta pesquisa teve o objetivo de analisar a atenção ao parto pelos enfermeiros dos hospitais públicos da ZL por meio da caracterização dos serviços e dos profissionais e da identificação das atividades Que eles desempenhavam e sua freqüência, bem como os possíveis obstáculos ou dificuldades Que encontravam para exercer sua função.

\section{MÉTODO}

Estudo descritivo, exploratório realizado em seis hospitais com leitos exclusivamente destinados ao Sistema Único de Saúde (SUS) da ZL no período de outubro de 2006 a junho de 2007.

A população foi composta por 62 enfermeiros Que atuavam na atenção ao parto nos hospitais selecionados. A amostra, por conveniência ou acidental, constituiu-se de todos aqueles que participaram da pesquisa preenchendo o instrumento de coleta de dados, o que correspondeu a 38 sujeitos, ou seja, $61 \%$ do universo de enfermeiros Que atuavam na assistência à mulher durante o trabalho de parto, parto e pós-parto imediato nos seis hospitais no período de coleta de dados.

Para primeiramente caracterizar a estrutura em Que ocorria a atenção à mulher no processo de parto e nascimento e, depois, ter acesso ao número de enfermeiros Que nela atuavam, foram entrevistados pelo pesquisador os dirigentes de enfermagem de cada um dos seis hospitais, após devida autorização pela direção das instituições e assinatura de Termo de Consentimento Livre e Esclarecido (TCLE).

Nessa entrevista foram utilizados formulários que continham Questões relativas à estrutura física e organizacional das maternidades, bem como o número de enfermeiros envolvidos na citada atenção. Ao final desse contato, era deixado para os enfermeiros um Questionário Que continha:

- Questões relacionadas à caracterização dos profissionais Quanto

à idade, formação, tempo de atuação na atenção pré-natal, composição da equipe de Que faziam parte e outros locais em Que trabalhavam;

- listagem de 83 atividades relativas à atenção ao parto acompanhada de escala ordinal com os atributos de freeüência nunca, Quase nunca, Quase sempre, sempre, na Qual os sujeitos pudessem assinalar Quais ações desenvolviam e com Que regularidade;

- espaço para Que indicassem por escrito acontecimentos, fatos ou eventos de ordem profissional ou institucional Que considerassem interferir ou servir de obstáculo para sua atuação na referida atenção.

Na confecção do Questionário, além da experiência pessoal da pesquisadora, foram utilizados documentos relacionados às competências profissionais em obstetrícia, à promoção à maternidade segura e às práticas obstétricas comumente utilizadas, bem como as recomendadas pelas evidências científicas ${ }^{(2-10)}$.

$\mathrm{O}$ referido instrumento foi disposto em envelope lacrado Que também continha carta de apresentação e de instruções, duas vias do TCLE e envelope selado e dirigido ao pesquisador em momento pré-estabelecido.

Todos os dados foram analisados por meio da estatística descritiva e as indicações de obstáculos foram categorizadas por similaridade temática. O projeto foi aprovado pelo Comitê de Ética em Pesquisa da Prefeitura de São Paulo (Parecer $n^{\circ}$. 0188/CEP/ SMS/2006) e todos os sujeitos receberam informações sobre a 
relevância da pesquisa, ficando-lhes livre a participação, de acordo com os TCLE.

\section{RESULTADOS}

A caracterização dos seis hospitais públicos da ZL mostrou Que realizavam em média 300 partos ao mês, dos Quais $27 \%$ eram cesáreas. A instituição Que tinha o menor índice de cesáreas, 15\%, foi vencedora do Prêmio Galba de Araújo em duas oportunidades, primeiro pelo modelo de atenção no Centro de Parto Normal (CPN) e depois pela Casa de Parto, anexa ao hospital que, à época de coleta de dados, apresentava média de apenas 15 partos ao mês, devido ao rígido protocolo estabelecido pela instituição para internação de mulheres naquele local, o Que resultou na menor demanda de parturientes, Que dificilmente eram encaminhadas da admissão médica para lá, e motivou diminuição de número de enfermeiros naquele setor.

Em Quatro hospitais, a atenção se dava exclusivamente em centros obstétricos tradicionais, ou seja, enfermarias conjuntas de pré-parto, algumas com cortinas entre os leitos, salas de parto com mesa obstétrica e macas para transporte e recuperação pós-parto. Nessas instituições, não eram utilizados protocolos de condutas alternativas para alívio da dor ou para realização de episiotomias, sendo comum a indicação de tricotomia genital, o uso de ocitócitos para aceleração do trabalho de parto, a restrição da parturiente ao leito, a liberação de acompanhantes para assistir apenas o nascimento, freqüentemente mediante autorização médica, e a aspiração rotineira de todos os recém-nascidos logo após o parto.

Nas outras duas instituições a atenção ocorria em CPN intrahospitalares e na Casa de Parto, todos com Quartos privativos no sistema PPP (pré-parto, parto e pós-parto imediato), o Que possibilitava a presença de acompanhante de escolha da mulher em todo o processo. Nesses locais, foi citado uso de protocolos para manejo da dor, restrição do uso rotineiro tanto de ocitócitos para condução do trabalho de parto euanto de episiotomia, possibilidade de escolha de posição não-litotômica para o parto e estímulo ao contato pele-a-pele e ao aleitamento na primeira hora do nascimento.

Verificou-se também Que em um dos seis hospitais as mulheres eram assistidas unicamente por profissionais médicos em todos os períodos do parto, ficando para as enfermeiras o gerenciamento do cuidado. Nessa instituição, Que não é referência para risco obstétrico, o índice médio de cesáreas ao mês chegava a 32\% e o partograma não era utilizado para o acompanhamento do progresso do trabalho de parto.

Nas demais cinco entidades, enfermeiros se responsabilizavam pela assistência e somente enfermeiras obstétricas assistiam 80 a $100 \%$ dos partos normais de baixo risco, ficando para os médicos a resolução de intercorrências, bem como a admissão de parturientes e o acompanhamento das puérperas.

Constatou-se que apenas os CPN e a Casa de Parto contavam com Quadro exclusivo de enfermeiras obstétricas. Nas outras instituições, os enfermeiros, nem todos com especialização em Obstetrícia, respondiam também pela atenção no alojamento conjunto e cobriam folgas ou ausências em outros setores do hospital.

A caracterização dos 38 participantes do estudo mostrou Que
40\% deles situavam-se na faixa etária dos 32 aos 41 anos; 58\% eram formados em Enfermagem há mais de 10 anos; 84\% eram especializados em Obstetrícia; $55 \%$ referiram ter participado de cursos de atualização na saúde materna e perinatal nos últimos cinco anos; $84 \%$ acompanhavam mulheres no pré-parto e realizavam partos; e $60 \%$ indicaram trabalhar em outras maternidades privadas ou públicas.

No item do Questionário destinado à indicação das atividades Que compõem a atenção ao parto, os sujeitos assinalaram Que realizavam sempre ou Quase sempre apenas $43 \%$ das 83 ações listadas, em porcentagens iguais ou maiores a $75 \%$. Algumas das restantes, mais significativas para discussão, e sua respectiva freqüência, são mostradas na Tabela 1 .

A maior parte (71\%) dos enfermeiros listou ou descreveu acontecimentos, fatos ou eventos de ordem profissional ou institucional Que serviam de obstáculo para sua atuação na atenção ao parto. A análise dessas indicações mostrou cinco categorias temáticas Que estão representadas na Tabela 2.

$\mathrm{Na}$ categoria "operacionalização da atenção" foram incluídos relatos de: sobrecarga de trabalho, maior número de médicos do Que enfermeiros por plantão, falta de valorização pelos dirigentes da instituição, baixa remuneração, salário não-diferenciado para as enfermeiras obstétricas, pagamento de produtividade ou de número de partos somente aos médicos obstetras, inadeeuação da estrutura física para a atenção humanizada, falta de material, de impressos e de protocolos assistenciais, proibições institucionais à atenção humanizada, falta de divulgação do trabalho, de atualização e de preparo pessoal ou da categoria.

Em "relação profissional" foram categorizadas as dificuldades no relacionamento dos sujeitos com os médicos obstetras e neonatologistas Que, segundo diversas descrições, não respeitam, não aceitam ou não consideram o trabalho das enfermeiras, utilizam condutas de forma arbitrária, não aceitam mudanças e insistem em procedimentos sem base científica, proíbem a presença de acompanhantes e não participam da assistência, deixando-a unicamente sob responsabilidade dos não-médicos.

Em "processo de trabalho", foram incluídas as descrições de sobrecarga ocasionada pela precariedade de recursos humanos, pelo remanejamento para outras áreas do hospital e pelo desgaste físico e emocional resultante das más condições de trabalho.

Em "atenção pré-natal", foram categorizados os relatos Que apontavam dificuldades na atenção decorrentes do inadequado preparo da mulher para o parto pelos serviços de pré-natal.

$\mathrm{Na}$ categoria "condições sócio-econômicas e culturais da população" peQuena parcela de enfermeiros indicaram Que os obstáculos decorrem da falta de participação ou compreensão pelas mulheres e acompanhantes de seu papel no processo de parto e nascimento.

\section{DISCUSSÃO}

A caracterização das instituições mostrou Que, na maior parte delas, as mulheres permanecem em trabalho de parto em espaços físicos limitados, o Que não possibilita privacidade e acompanhantes de livre escolha, em detrimento do Que determina legislação federal, exigível e aplicável em todo o país ${ }^{(12)}$. Nessas instituições, também foram indicadas condutas desaconselhadas pelas evidências 
Tabela 1. Atividades realizadas com freeüência menor Que 75\% pelos enfermeiros na atenção ao parto. São Paulo, 2008.

\begin{tabular}{|c|c|c|}
\hline \multirow{2}{*}{ Atividades } & \multicolumn{2}{|c|}{ FreQüência } \\
\hline & $n$ & \% \\
\hline Admissão de parturientes por meio de anamnese e exame clínico obstétrico & 24 & 63 \\
\hline Indicação de instalação venosa rotineira no momento da admissão da parturiente & 20 & 53 \\
\hline Avaliação e controle da eliminação urinária durante o trabalho de parto & 22 & 58 \\
\hline Utilização de ocitócitos para condução ou aceleração do trabalho de parto & 20 & 53 \\
\hline Prescrição e uso de métodos não-farmacológicos para alívio da dor durante o trabalho de parto & 27 & 70 \\
\hline Estímulo à realização de "puxos" na dilatação completa ou Quase completa & 27 & 70 \\
\hline Orientação ao "puxo" prolongado e dirigido durante o expulsivo & 27 & 70 \\
\hline Utilização de posição não litotômica no período expulsivo & 23 & 61 \\
\hline Uso rotineiro de posição litotômica com ou sem estribos no expulsivo & 24 & 63 \\
\hline Aspiração e/ou lavagem gástrica rotineira dos recém-nascidos & 12 & 34 \\
\hline
\end{tabular}

Tabela 2. Categorização das dificuldades indicadas pelos enfermeiros para a atenção ao parto. São Paulo, 2008.

\begin{tabular}{llcc}
\hline & Categorias temáticas & \multicolumn{2}{c}{ Dificuldades } \\
\hline 1. & Operacionalização da atenção & $\boldsymbol{n}$ & 44 \\
2. & Relação profissional & 50 & 21 \\
3. & Processo de trabalho & 24 & 18 \\
4. & Atenção pré-natal & 20 & 3 \\
5. & Condições sócio-econômicas e culturais da população & 3 \\
\hline
\end{tabular}

científicas, como a condução do trabalho de parto com ocitócitos e a utilização rotineira de episiotomia.

Os dois hospitais com CPN e Casa de Parto, únicos credenciados pela Iniciativa Hospital Amigo da Criança, se destacaram por privilegiar o conforto e a maior participação da mulher, com apoio e presença de acompanhantes, em ambiente mais agradável e acolhedor, Que consegue reduzir a ocorrência de partos operatórios, diferentemente do Que ocorre nos centros obstétricos tradicionais. São essas as entidades Que instituíram mudanças na atenção ao parto e nascimento, tornando-a mais humanizada e adeQuada às recomendações comprovadamente benéficas ${ }^{(7-10)}$, haja vista os prêmios recebidos por uma delas.

A caracterização dos participantes delineou a especificidade e a realidade do campo de trabalho na atenção à maternidade, ou seja, a existência de peQueno número de enfermeiras obstétricas que atuam em várias instituições, por necessidade financeira e pela demanda por profissionais experientes, realidade imposta pelo baixo nível salarial, notadamente no setor público hospitalar, características marcantes do mercado de trabalho dos enfermeiros na América Latina ${ }^{(13,14)}$.

Em relação às práticas obstétricas, observa-se Que a alta porcentagem (53\%) de instalação rotineira de venóclise no momento da internação da mulher no pré-parto pode ter efeitos indesejados, como hiponatremia materno-fetal e hipoglicemia neonatal ${ }^{(7)}$, além de contribuir para a restrição da mulher ao leito, outra prática não recomendada durante o trabalho de parto, mas confirmada pela inadequada estrutura física da maior parte dos hospitais pesquisados.

$\mathrm{O}$ uso rotineiro do partograma, assinalado por $81 \%$ dos enfermeiros, é uma das tecnologias indicadas pelas evidências científicas para a monitorização do trabalho de parto ${ }^{(4-10)}$, inexistente unicamente na instituição em Que só os médicos acompanhavam o processo de parto e nascimento.

Quanto à condução do trabalho de parto com ocitócitos, ação indicada por $53 \%$ dos sujeitos, as evidências científicas apontam Que essa conduta nunca deve ser rotineira, primeiro por restringir a movimentação e deambulação da mulher e, segundo, por contribuir para efeitos colaterais, como taquisistolia, hipertonia, hiperestimulação uterina, rotura uterina e sofrimento fetal associado $^{(4-10)}$.

A movimentação da parturiente durante o trabalho de parto tem várias vantagens, incluindo aumento das contrações Que ajudam a dilatação cervical, maior conforto materno e diminuição do uso de analgésicos, o Que não ocorre em ambientes sem espaço e sem condições de propiciar conforto. A importância da presença do acompanhante, Que dá apoio, ouve, estimula, massageia e conforta a gestante, associa-se à menor necessidade de analgesia, parto operatório e experiências não satisfatórias, bem como aumenta o índice de aleitamento materno e diminui a ocorrência de depressão puerperal $^{(7-10)}$.

Ambientes desfavoráveis, sem espaço para deambulação, sem a possibilidade de presença de acompanhantes, nos Quais a gestante permanece continuamente deitada, prolongam o trabalho de parto e causam aumento do uso de ocitócitos para estimular as contrações, Quadro que condiz com o Que foi indicado por mais da metade dos sujeitos e pelos dirigentes dos Quatro hospitais Que não contavam com centros de parto normal intra ou extra-hospitalares.

O estímulo aos "puxos" maternos, esforços voluntários de expulsão, 
longos e dirigidos, geralmente acompanhados de apnéia forçada pela manobra de Valsalva e interrompidos pela respiração profunda, foi assinalado por mais de $70 \%$ dos enfermeiros. Esta prática predispõe a alterações na freeüência cardíaca fetal e redução do índice de Apgar, especialmente Quando a mãe está em decúbito dorsal, posição associada à compressão da aorta distal e à redução do fluxo sanguíneo para o útero e membros inferiores, o Que produz alterações circulatórias maternas e comprometimento da oxigenação fetal. Por este motivo, a recomendação é permitir Que a mulher durante o período expulsivo faça os esforços de puxo mais curtos e de forma espontânea, preferentemente em posições verticalizadas ${ }^{(4-10)}$.

Vale ressaltar ainda Que o "puxo" espontâneo pode ser inibido por ambientes desfavoráveis e hostis, pela posição litotômica para o parto ou pela bexiga estar cheia, sendo, por isso, importante monitorar a eliminação urinária durante o primeiro período do parto $^{(8,10)}$. Este cuidado, indicado por apenas $58 \%$ dos enfermeiros, deve ser redobrado Quando as mulheres permanecem por longo tempo deitadas, com infusões venosas rotineiras, o Que ocorria na maior parte das instituições pesquisadas.

O uso da posição litotômica dorsal da mulher para o parto, apontada por $63 \%$ dos sujeitos, é outra conduta Que se associa ao aumento do segundo período do parto, à maior taxa de intervenções Que restringem a participação da mulher, como puxos controlados e manobras de auxílio à expulsão, às alterações da freqüência cardíaca fetal por compressões circulatórias e intervenções como a episiotomia $^{(4-10)}$. A recomendação consensual é de evitar a posição horizontal durante o período expulsivo; desse modo, a mulher assume a posição Que preferir e os profissionais devem respeitá-la, estando treinados para isso ${ }^{(8)}$.

A aspiração rotineira de todos os recém-nascidos logo após o parto também é procedimento a ser Questionado, havendo evidências científicas de que a maioria dos bebês saudáveis não requer aspiração nasal e faríngea, muito menos gástrica. Caso seja necessária limpeza das vias aéreas, ela deve ser feita com peras de aspiração, em vez de cateteres, Que podem provocar desde lesões na mucosa até arritmias cardíacas. Como na maioria dos partos de baixo risco os bebês são saudáveis, recomenda-se Que estes sejam avaliados e cuidados junto à sua mãe. Isto ocorrerá no colo materno, durante o contato pele-a-pele, sendo importante nesse momento apenas um cuidador vigilante Que promova calor, vias aéreas livres e recepção amistosa ${ }^{(7)}$.

Em detrimento de todas essas observações, verificou-se Que o conjunto de atividades assinaladas pelos enfermeiros Qualifica a atenção e dá valor ao trabalho Que realizam, sendo possível inferir Que sem sua participação, a atenção ao parto resultaria em piores indicadores de Qualidade assistencial materna e perinatal como, por exemplo, altos índices de intervenções e de partos cirúrgicos, haja vista os 32\% de cesáreas no único hospital em Que os partos são assistidos apenas por médicos.

Pela análise dos obstáculos relatados, observa-se Que os enfermeiros estão alinhados com os preceitos da humanização da assistência ao parto, sendo, todavia, impedidos institucional ou profissionalmente a fazê-lo. Entretanto, a descrição da atenção em cada um dos hospitais e a análise das atividades assinaladas mostrou Que eles também são responsáveis pela medicalização do parto na medida em Que utilizam práticas tradicionais e desaconselhadas pelas evidências científicas, muitas delas prejudiciais à saúde materna e perinatal, conforme exposto.

A respeito de sua difícil relação com os médicos, ressalta-se Que, segundo diversos autores ${ }^{(8,15-17)}$, desmedicalizar o parto implica em perda de poder; abandonar rotinas que adéQuam o trabalho de parto ao modo de funcionamento do hospital, adotando outras Que privilegiam o acompanhamento de sua fisiologia, significa perda de controle. Como essas mudanças retiram a referência do papel médico no parto, ocorre resistência desses profissionais, em detrimento, até, da Qualidade assistencial e do respeito aos direitos humanos.

Caso realmente a gestante, ao conhecer seus direitos, tivesse condições de exigi-los dos serviços de saúde, muito do Que foi exposto, não aconteceria. Tendo em vista as reais e precárias condições sócio-culturais de nossa população, o ideário da humanização da assistência, Que pressupõe mulheres sujeitos do processo, exige muito mais do Que mudanças dos modelos de atenção. A prática mostra Que grande parte das mulheres não tem noção sequer do Que seja respeito à sua individualidade, satisfazendo-se tão somente com a existência de um leito obstétrico para parir. Também, Que não é protagonista do processo, o Que resulta na valorização da intervenção e na hegemonia médica ${ }^{(17,18)}$.

Por conseguinte, mulheres e acompanhantes, precisam conhecer seus direitos a fim de se defender de práticas abusivas, desnecessárias, discriminatórias, iatrogênicas, sem base científica, porém, utilizadas no dia-a-dia da assistência à saúde materna e perinatal em nosso país ${ }^{(16,18)}$. Não cabe ao profissional de saúde aceitar ou não a presença do acompanhante, ela é obrigatória, é um direito conquistado pelas mulheres.

Pelo visto, a lei em si não é segurança para a implementação desta medida ou de outras que o Ministério da Saúde insiste em recomendar e apoiar, como a diminuição do índice de cesáreas, a inclusão de maior número de enfermeiras obstétricas e obstetrizes na atenção pública e privada ao parto, a mudança dos ambientes hospitalares para maior comodidade e segurança à mulher, entre outros.

A re-organização dos serviços públicos depende dos gestores, nem sempre afinados com as políticas públicas e cobrados pelos órgãos responsáveis, pela população e, até, pelos profissionais de saúde. Conforme descrito, as evidências científicas não são suficientes para a mudança de um modelo assistencial intervencionista, medicalizado e não centrado na mulher.

A "vitimização da mulher pelo parto violento", ainda freeüente nos serviços públicos, e a sua transformação em "objeto ou propriedade”, em decorrência da institucionalização do parto, constituem-se em práticas incompatíveis com a humanização, modelo Que exige desde redimensionamento dos papéis e poderes no cenário da assistência ao parto e nascimento até a prática profissional competente $e^{(16,18,19)}$.

Diante do exposto, das ações assinaladas pelos sujeitos e, principalmente, dos obstáculos descritos, pôde-se ter um Quadro de como ocorre a atenção ao parto por enfermeiros na ZL. Os resultados apresentados mostram Que os enfermeiros não dispõem de meios para operacionalizar a assistência, por enfrentarem o poder de decisão do médico na determinação de condutas contrárias à humanização e às evidências científicas, por se defrontarem com o excesso de trabalho decorrente da precariedade de recursos humanos ou da falta de colaboração, por não se sentirem valorizados pelos serviços públicos de saúde, por não contarem, enfim, com 
condições dignas de trabalho e salários gratificantes.

\section{CONCLUSÕES}

Como o exercício de uma profissão não se limita ao âmbito de sua aplicabilidade, exige profundidade de conhecimentos, saberes e, principalmente, exercício reflexivo, por meio do eual os sujeitos mobilizam um conjunto de valores, aprendizagens e habilidades em favor das situações, conclui-se Que para Que os enfermeiros da ZL colǫuem em prática a atenção Qualificada ao parto, eles devem contar com estruturas favoráveis e com o fortalecimento de sua identidade profissional, o Que pressupõe maior poder e autonomia ${ }^{20 .}$ ${ }^{21)}$, não sendo esta a condição descrita neste estudo.

De forma semelhante a outro trabalho ${ }^{(13)}$, os achados desta pesquisa mostram que o modelo de cuidado da maior parte dos hospitais públicos pesquisados se constrói muito mais pelas posições hierárQuicas do Que pela Qualificação profissional ou pelas recomendações da comunidade científica, ou seja, os enfermeiros ainda são impedidos de atuar com autonomia, competência e Qualidade pela desvalorização de sua capacidade profissional.

Mesmo com a limitação de não serem avaliados desempenhos profissionais, a identificação das ações relacionadas a eles e, especialmente, dos obstáculos à sua execução, possibilitou visualizar as barreiras Que impedem a atenção Qualificada ao parto na região pesquisada.

De acordo com documento Que valoriza e prioriza o trabalho das enfermeiras obstétricas e das obstetrizes na promoção da maternidade segura ${ }^{(21)}$, os obstáculos Que impedem a atenção Qualificada por estas profissionais relacionam-se ao desconhecimento de sua capacidade pelos sistemas de saúde, seu número insuficiente, sua falta de atualização teórica e clínica, a falta de fundos para sua capacitação ou educação permanente, sua baixa remuneração, os conflitos e rivalidades que enfrentam com os médicos, a falta de incentivo e de políticas de incremento de recursos humanos que os impedem de trabalhar de forma segura, sua falta de união e de fortalecimento profissional e, principalmente, o não estabelecimento por eles de parcerias com as mulheres a fim de gozar de maior reconhecimento.

Muitos desses problemas foram salientados pelos participantes desta pesquisa, parecendo claro oue mudar esse Quadro demanda Que as estruturas públicas de São Paulo revisem suas políticas de modo a garantir a implementação dos programas já existentes e das diretrizes do SUS no Que se refere tanto à melhoria da atenção materno-infantil Quanto à valorização e, de forma especial, à inclusão de maior número de enfermeiras obstétricas e obstetrizes na atenção ao parto.

\section{REFERÊNCIAS}

1. Lansky S, França E, Leal MC. Mortalidade perinatal e evitabilidade: revisão da literatura. Rev Saúde Pública 2002 36(6): 759-72.

2. World Health Organization (WHO). Making pregnancy safer: the critical role of the skilled attendant. Geneva: WHO; 2004.

3. International Confederation of Midwives (ICM). New York: ICM; [cited 2006 Aug 28]. Competencies; [about 12 screeens]. Available from: http://www.internationalmidwives.org/

4. World Health Organization (WHO). Safe motherhood needs assessment. Geneva: WHO; 2001 .

5. Organização Mundial da Saúde (OMS). Maternidade segura. Assistência ao parto normal: um guia prático. Genebra: OMS; 1996.

6. Ministério da Saúde (BR). Parto, aborto e puerpério: assistência humanizada à mulher. Brasília (DF): Ministério da Saúde; 2001 .

7. Enkin M, Keirse MINC, Neilson J et al. Guia para atenção efetiva na gravidez e no parto. $3^{\text {a }}$ ed. Rio de Janeiro: Guanabara Koogan; 2005.

8. Cecatti JG, Calderón IMP. Intervenções benéficas durante o parto para a prevenção da mortalidade materna. Rev Bras Ginecol Obstet 2005; 27(6): 357-65.

9. Hofmeyr G). Evidence-based intrapartum care. Best Practice \& Research Clinical Obstetrics and Gynaecology 2005; 19(1): 103-15.

10. Intrapartum care: care of healthy women and their babies during childbirth - Clinical Guideline. London: Royal College of Obstetricians and Gynaecologists Press; 2007.

11. Secretaria Municipal de Saúde (SMS). Boletim CEInfo 2006. São Paulo (SP): SMS; 2006.

12. Ministério da Saúde (BR). Lei no I 1 . 108. Altera a Lei nº 8.080, de 19 de setembro de 1990, para garantir às parturientes o direito à presença de acompanhante durante o trabalho de parto, parto e pós-parto imediato, no âmbito do Sistema único de Saúde - SUS. Publ. Diário Oficial da União 2005.

13. Dotto LMG. Atenção Qualificada ao parto: a realidade da assistência de enfermagem em Rio Branco (AC) [tese]. Ribeirão Preto: Escola de Enfermagem de Ribeirão Preto/USP; 2006.

14. Malvárez SM, Castrillón MCA. Overview of the nursing workforce in Latin America. Washington: PAHO; 2005.

15. Dias MAB, Domingues RMSM. Desafios na implantação de uma política de humanização da assistência hospitalar ao parto. Cien Saúde Coletiva 2005; I0(3): 669-705.

16. Diniz CSG, Chacham AS. O "corte por cima" e o "corte por baixo": o abuso de cesáreas e episiotomias em São Paulo. Questões Saúde Reprod 2006; I (1): 80-91.

17. Diniz CSG. Humanização da assistência ao parto no Brasil: os muitos sentidos de um movimento. Cien Saúde Coletiva 2005; 10(3): 627-37.

18. Teixeira NZF, Pereira WR. Parto hospitalar: experiências de mulheres da periferia de Cuiabá-MT. Rev Bras Enferm 2006; 59(6): 740-4.

19. Castro IC, Clapis MI. Parto humanizado na percepção das enfermeiras obstétricas envolvidas com a assistência ao parto. Rev Latino-am Enfermagem 2005; 13(6): 960-7.

20. Corbellini VL, Medeiros MF. Fragmentos da história: a enfermeira tornando-se sujeito de si mesma. Rev Bras Enferm 2006; 59(esp): 397-402.

21. United Nations Population Found (UNFPA). Expectation and delivery: investing in midwives and others with midwifery skills. New York: UNFPA; 2007. 